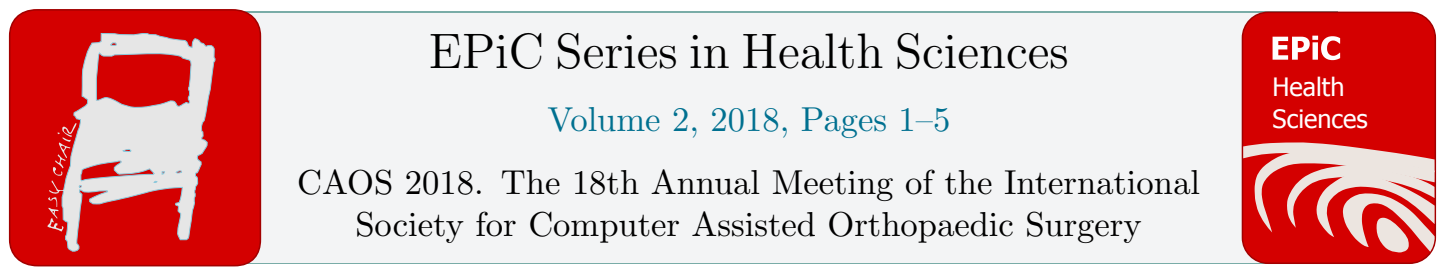

\title{
5-year gait analysis as a secondary outcome of a fixed bearing robotic assisted versus mobile bearing manual UKA RCT
}

\author{
Matthew Banger ${ }^{1}$, Philip Rowe ${ }^{1}$, Lindsay Millar ${ }^{1}$, Mark Blyth ${ }^{2}$, Angus \\ MacLean $^{2}$, Bryn Jones ${ }^{2}$ and Iona Donnelly ${ }^{2}$ \\ ${ }^{1}$ University of Strathclyde, Glasgow, UK \\ ${ }^{2}$ Glasgow Royal Infirmary, GGCNHS, Glasgow, U.K. \\ matthew.banger@strath.ac.uk
}

\begin{abstract}
Total knee arthroplasty (TKA) procedures have recognised differences in knee kinematics and kinetics from healthy knees. This study reports on the less documented knee kinematics and kinetics of uni-compartmental knee arthroplasty (UKA) patients during walking as a secondary outcome measure of a RCT between a fixed bearing UKA robotic procedure versus a mobile bearing UKA manual procedure.

Three dimensional, instrumented, gait analysis was performed 5 years after 49 medial osteoarthritis UKA patients received surgery. 27 were randomised to receive a robotic assisted UKA and 22 to receive a manual implant surgery.

The robotic assisted group showed a significant increased range of motion in stance during walking. 59\% of the robotic assisted group achieved a bi-phasic sagittal knee moment, in comparison of $50 \%$ of the manual group. Significantly higher peak values of the varus knee and internal rotation moments were measured for the robotic assisted group during early and late section of the gait cycle, respectively. This indicates that larger normalised forces are applied through the knee during flat ground walking in the robotic assisted group.

The robotic assisted group showed biomechanical benefits over the manual group for walking tasks. Poor fixation outcomes are avoided in patients with normal knee moments.
\end{abstract}

\section{Introduction}

It is thought that patients who desire a restoration of lifestyle, minimised surgery, reduced pain and rapid recovery may benefit from uni-condylar knee arthroplasty. Knee kinematics and kinetics in total knee arthroplasty (TKA) patients have consistently demonstrated poorer outcomes than aged matched controls (Rowe et al., 2007). Additionally, knee biomechanics have been shown to be closer to normal 
values in computer assisted TKA procedures (McClelland et al., 2017). In a comparative gait analysis between uni-compartmental knee arthroplasty (UKA) and TKA, Chassin et al have demonstrated that a greater percentage of UKA patients (70\%) maintained a normal biphasic flexion/extension moment pattern about the knee when compared with similar groups of TKA patients (Chassin et al., 1996). It is therefore the aim of this study to further the understanding of kinematics and kinetics in UKA patients performing functional tasks.

By performing a scientific, precise, accurate and multi-dimensional assessment of patient biomechanical performance, functional outcome, activity and satisfaction it will be possible to determine the benefits (if any) of a robotic system over a conventional system.

Previously reported outcomes from this study have shown robotic-assisted surgical procedures led to improved accuracy of implant positioning compared with conventional unicompartmental knee arthroplasty surgical techniques. Additionally, robotic arm-assisted surgery resulted in improved early pain scores and early function scores (3 months post-op) in some patient-reported outcomes measures, but no difference was observed at one year post-operatively.

\section{Methodology}

In total, 49 patients treated with UKA for isolated medial osteoarthritis completed the functional assessment at 5 years: 27 in the Robotic assisted UKA patient group and 22 in the manual UKA group. The robotic assisted group received a fixed bearing Restoris MCK unicondylar knee replacement implanted with the aid of the Mako System, a tactile guided robotic arm-assisted system. The manual group received a conventional mobile bearing Oxford partial knee operation carried out using standard manual instrumentation and the Oxford Phase 3 UKA. Patients were randomised into either group and surgery was performed by senior surgeons that regularly used the Oxford procedure, but new to the MAKOplasty procedure. The study was powered for the primary outcome of a 1 degree difference in CT measurement of the tibial sagittal slope at one year, $n=150.126$ reached 1 year, and 5 years followup is still being conducted. No functional differences were shown pre-operative between the groups in either the median pre-operative UCLA (IQR) robotic 4 (3 to 6), manual 3.5 (3 to 5) $\mathrm{p}=0.430$ (t-test) or Mean SF-12 (Physical Component Score) (SD) robotic 32.8 (7.7) manual 32.5 (8.3) p=0.837 (t-test).

The outcome measure was the peak moments in flexion, adduction and internal rotation at the knee. Biphasic sagittal knee moments were determined as an average knee moment profile presenting positive peaks in the first and last halves of the gait cycle that flank a negative peak in the middle half of the stance phase of the gait cycle.

\subsection{Testing protocol}

Patients were assessed for knee movements and moments while performing a 10 metre walking task. Patients were asked to walk at a comfortable walking speed while motion capture equipment (12 camera VICON bonita system, 2 AMTI Gen5 forceplate) recorded movements and ground reaction forces. At least three clean heel strikes were recorded on the force plates. Strathclyde cluster biomechanical model was used to calculate the resulting joint kinematics and internal moments. All moments were normalised for patient weight and leg length. Testing was conducted in the Human Performance Laboratory in clinical research facility at the Glasgow Royal Infirmary (UK). 


\section{Statistical Analysis}

Statistical differences were calculated with a Mann-Whiteney $U$ test to compare continuous variables that were not normally distributed, or independent t-tests if normally distributed $(\alpha=0.05)$.

\subsection{Data processing}

A Strathclyde cluster model was used to calculate the Biomechanical outputs that was performed in VICON Nexus (ver 2.6). All subsequent analysis was completed using MatLab (R2016b $(9.1 .0 .441655))$.

\section{Results}

The robotic assisted patient group showed a significant increase in the range of motion from early peak flexion to mid-stance (3.98 degrees, $\mathrm{p}=0.002$ ).

Kinetically, $59 \%$ of the robotic assisted group and $50 \%$ of the manual group presented a bi-phasic knee moment. The coronal knee moment showed a significant difference $(\mathrm{p}=0.027)$ in the peak varus moment at early/mid stance, and a further difference but this was not significant at late stance $(\mathrm{p}=0.11)$ (Figure 1).

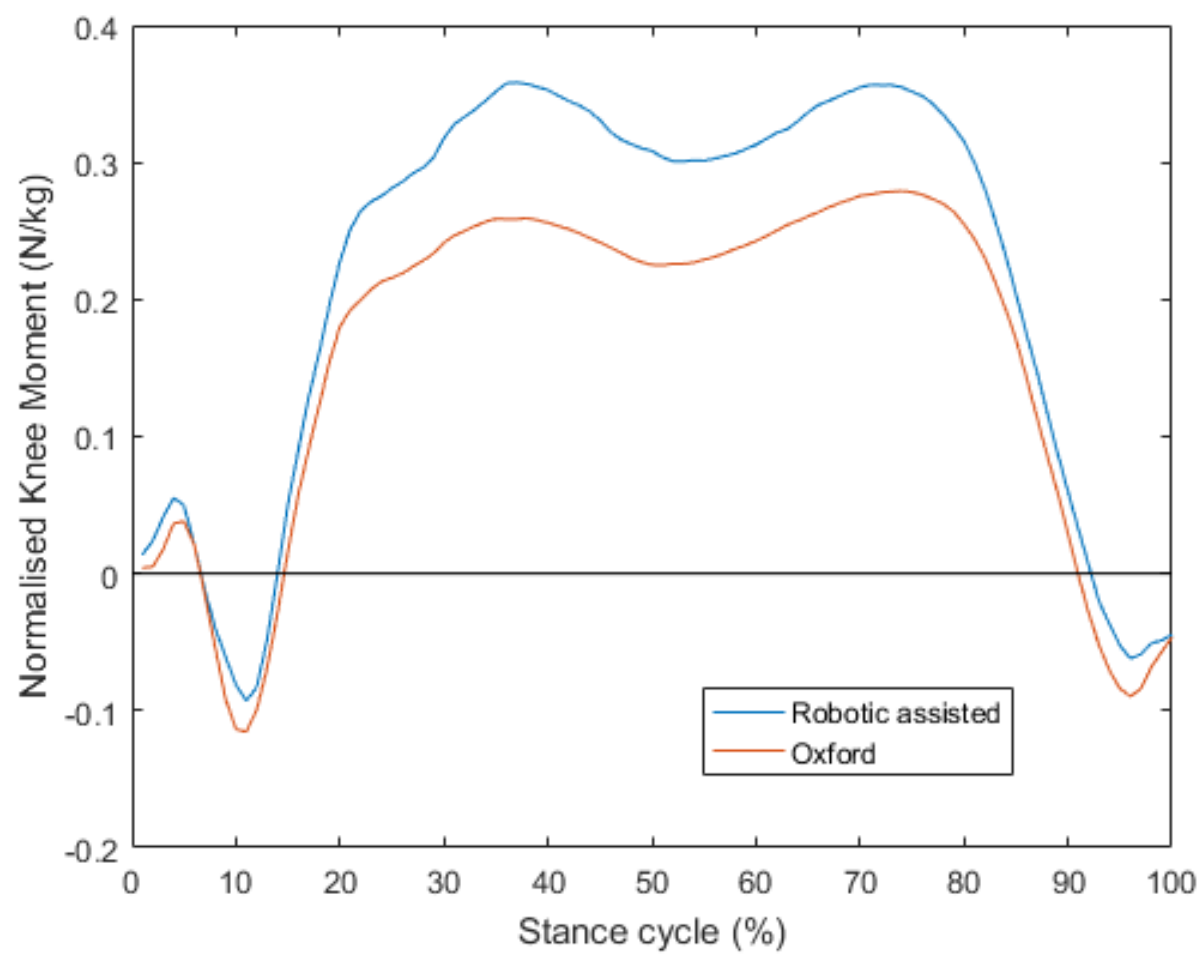

Figure 1 Varus Knee Moment. The initial peak has a difference of $0.10, p=0.027$, the second peak has a difference of $0.07, p=0.11$. 
The transverse knee moment showed a significant difference $(\mathrm{p}=0.05)$ in the peak internal rotation moment at late stance, during push off (Figure 2).

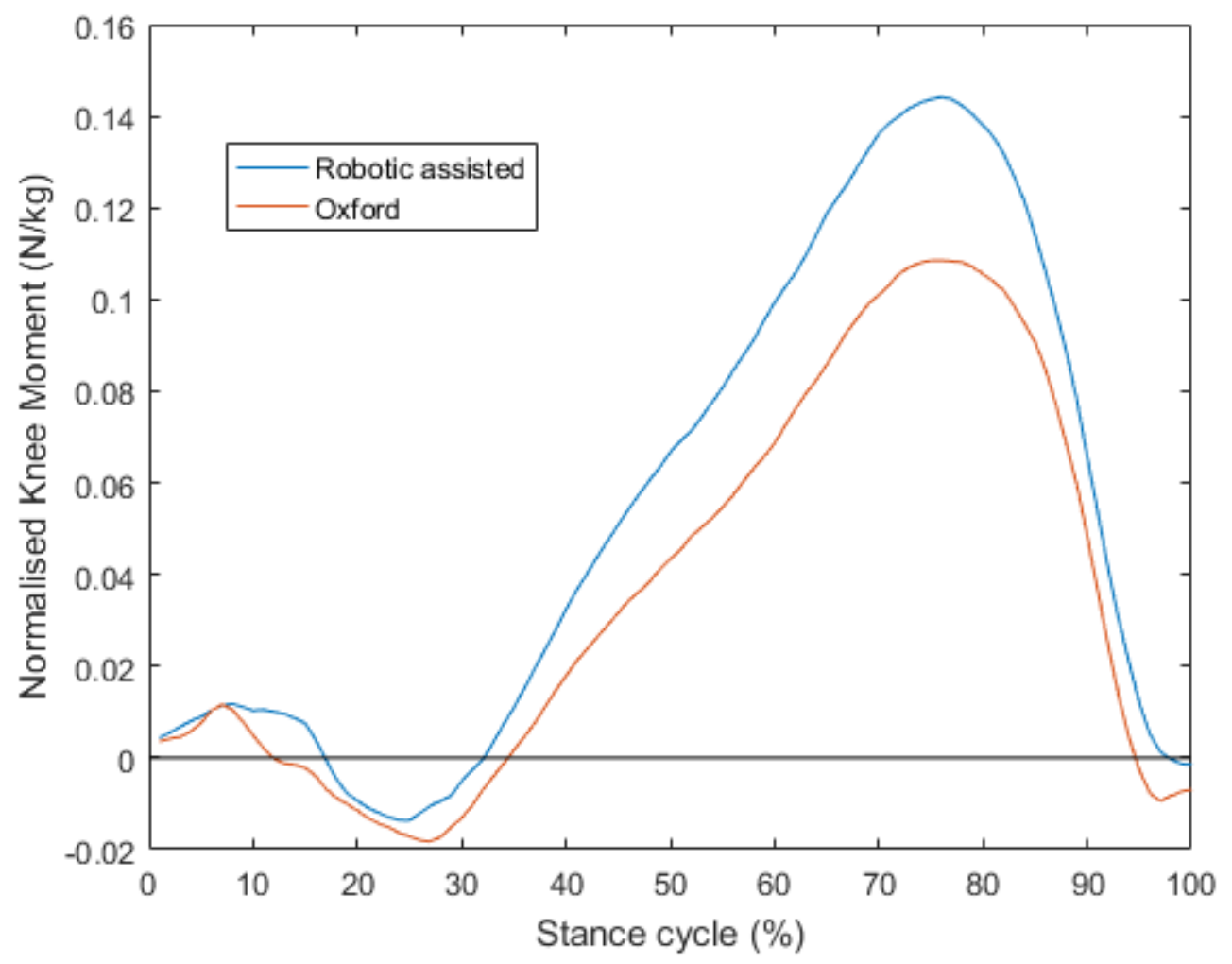

Figure 2 Internal Moment. Difference at the peak was $0.04, p=0.05$

\section{Discussion}

Differences in the kinematics of the knee were shown, as was previously reported at 1 year and these were similarly significant at 5 years. Gait analysis is sensitive to differences in the functionality of the knee, but with the addition of force measurements these can show significant differences in walking patterns. Biphasic sagittal knee moments are a dichotomous measure of normal gait. The robotic assisted group showed a 9\% higher presentation of bi-phasic gait. While the significance of this difference is hard to determine, the robotic group presented a higher percentage of patients with dichotomous 'normal' gait. However, as presented from Chassin (Chassin et al., 1996), the preoperative function of the knee is a factor in determining if biphasic knee moments are to be presented post-operatively. Pre-operative analysis was not performed during this study. Poor post-operative TKA tibal fixation of the implant has been associated with abnormal knee flexion moments (Hilding et al., 1999), suggesting that poor outcomes can be avoided by restoring normal knee moments.

Larger coronal moments are a result of larger lateral forces in the knee. The moments were similarly larger in the axial plane; however, this was at a later stage of stance. Larger forces and moments, normalised for height and weight, being applied through the knee would require a stable knee. These forces initially stop the knee collapsing during weight acceptance and allow propulsion of the body at 
the later stages of stance. While stability of the knee could not be determined for this study, the robotic assisted group applied larger force through their knee during flat ground walking at both weight acceptance and late stance.

Implant design needs to be considered in these two groups, with the robotic assisted group receiving a fixed bearing implant while the manual group received a mobile bearing. The mobile bearing, while allowing for greater surgical tolerances allows articulation of the tibial insert both laterally and rotationally over a flat metal surface, resulting in lower resistive forces. For this reason, the differences in the moments are a result of implant design as much as surgical procedure.

\section{Reference}

Chassin, E.P., Mikosz, R.P., Andriacchi, T.P., Rosenberg, A.G., 1996. Functional analysis of cemented medial unicompartmental knee arthroplasty. J. Arthroplasty 11, 553-559.

Hilding, M.B., Ryd, L., Toksvig-Larsen, S., Mann, A., Stenström, A., 1999. Gait affects tibial component fixation. J. Arthroplasty 14, 589-593.

McClelland, J.A., Webster, K.E., Ramteke, A.A., Feller, J.A., 2017. Total knee arthroplasty with computer-assisted navigation more closely replicates normal knee biomechanics than conventional surgery. The Knee 24, 651-656. https://doi.org/10.1016/j.knee.2016.12.009

Rowe, P.J., Myles, C.M., Nutton, R.W., 2007. knee arthroplasty on joint movement during functional activities and joint range of motion with particular regard to higher flexion users. Journal of Orthopaedic Surgery, 13 (2). pp. 131-8. ISSN 1022-5536. 